\title{
Quantitative proteomics reveals distinct molecular signatures of different cerebellum-dependent learning paradigms
}

Yong Gyu Kim ${ }^{1,2}$, Jongmin Woo ${ }^{1,5}$, Joonho Park ${ }^{3}$, Sooyong Kim ${ }^{1,2}$, Yong-Seok Lee ${ }^{1,2,4}$, Youngsoo $\mathrm{Kim}^{1,3, *}$, Sang Jeong $\mathrm{Kim}^{1,2,4}$ *

\begin{abstract}
Affiliations
${ }^{1}$ Department of Biomedical Sciences, Seoul National University College of Medicine, Seoul 03080, Korea

${ }^{2}$ Department of Physiology, Seoul National University College of Medicine, Seoul 03080, Korea

${ }^{3}$ Interdisciplinary Program in Bioengineering, College of Engineering, Seoul National University, 1 Gwanak-ro, Seoul 151-742, Korea

${ }^{4}$ Neuroscience Research Institute, Seoul National University College of Medicine, Seoul 03080, Korea

${ }^{5}$ Environmental Molecular Sciences Laboratory, Pacific Northwest National Laboratory, Richland, Washington 99354, United States
\end{abstract}

*Corresponding Authors

E-mail address: biolab@snu.ac.kr (Youngsoo Kim), sangjkim@snu.ac.kr (Sang Jeong Kim) 

learning groups with memory consolidation of 24 hour

*Supplemental Table S1. Protein discovery table (XSLX)

${ }^{*}$ Supplemental Table S2. Protein quantification of individual samples (5,347 proteins) (XSLX)

*Supplemental Table S3 (a-b). Protein set enrichment and clustering analysis (XSLX)

*Supplemental Table S4 (a-h). Weighted gene co-expression analysis (WGCNA) (XSLX)

$\left({ }^{*}\right.$ submitted as separate files) 


\section{Supplemental Methods}

Real-time quantitative PCR. Expression level of transcripts of target proteins was quantified in Home$\mathrm{Ctrl}(\mathrm{n}=3)$ and LTM of OKR-up $(\mathrm{n}=3)$ groups by real-time (RT) quantitative PCR (qPCR). Cerebellar flocculi were obtained from 7-12 weeks old mice $(n=3)$. Total RNA was purified from the collected sample using the RNeasy Mini Kit (QIAGEN) according to the manufacturer's instructions. Isolated total RNA was quantified by Nanodrop (ThermoFisher). The same amount of total RNA per sample was reverse transcribed into cDNA using SuperScript III First-Strand (Invitrogen). RT qPCR was conducted using the CFX Connect system (Bio-Rad) with primers selected from the Harvard Primer Bank (http://pda.mgh.harvard.edu/primerbank/index.html). Primer sequences are available upon request. Mean Ct values were used relative quantification of mRNA expression between Home-Ctrl and OKR-up group using the Pfaffl's (2001) method[1], with the ratio of the target gene expressed relative to the mean of Actb ( $\beta$-actin).

\section{Supplemental Text}

Itpr1 (Inositol 1,4,5-trisphosphate receptor 1) is a class of intracellular $\mathrm{Ca} 2+$ channels mediating the release of $\mathrm{Ca} 2+$ from intracellular stores[2], which is highly expressed in cerebellar Purkinje cells[3]. Previous studies have shown that the cerebellar LTD impairment was present in Itpr1-knockout mice[4]. Therefore, the increase in Itpr 1 at $1 \mathrm{~h}$ after VOR-dn learning is likely to be related to intracellular Ca2+ dynamics in Purkinje cell (PC) and the cerebellar LTD.

Psen1 (Presenilin 1) is the catalytic core $\gamma$-secretase complex, and closely related to Alzheimer's disease[5]. It was suggested that Psen1 is involved in regulation of neurotransmitter release and synaptic plasticity in the hippocampus [5, 6]. Given the presence of Psen1 in cerebellar granule cells (GCs) and PCs[7], we speculate that an alteration in synaptic vesicle release may occur in GCs or PCs in forming STM of OKR-up.

Syn 2 (Synapsin 2) is a presynaptic protein interacting with synaptic vesicle[8]. In contrast to the broad expression of Syn 1 in axon terminal of GCs, Syn 2 is restricted to a subpopulation of GC boutons[9], which was downregulated in STM group of OKR-up in our study. Previous study suggested that the heterogeneous expression of Syn 2 may affects short-term plasticity between GC and interneuron (IN) and, consequently, the excitation-inhibition balance in neural networks[9]. Our results suggest that synaptic plasticity between GC and IN may be engaged at $1 \mathrm{~h}$ after VOR-dn learning.

Cplx2 (Complexin 2) is known to be localized in the presynaptic terminal, and to modulate neurotransmitter release[10, 11]. Cplx2 knockout mice showed deficient mossy fiber-CA3 LTP in the hippocampus and cognitive deficits in the Morris water maze[12,13]. Since Cplx2 is known as a reliable marker of excitatory presynaptic terminal in a brain[14], the functional contribution of this molecule would be expected at the terminal of GC, the most abundant excitatory neuron in the cerebellum, to mediate synaptic transmission to PC and IN.

Stmn1 (Stathmin 1) is a cellular remodeling protein regulating the disassembly of microtubules, an essential structural component of dendrites[15-17]. Stmn1 is known to play as a regulator of fear memory, and to be required for the induction of synaptic plasticity in the amygdala[18]. This protein is of particular interest because it's functional role in cerebellar PCs has been suggested. Ohkawa $\mathrm{N}$ et al (2007) showed that Stmn1 regulates Ca2+-dependent development of dendritic arborization during neuronal development of PC[19]. Given the previous suggestion that LTM of OKR-up might be attributed to structural remodeling of synaptic connections of PCs[20], it is likely that increased Stmn1 at $24 \mathrm{hrs}$ after OKR-up learning decreased the number of synaptic connections in PCs.

Snca ( $\alpha$-synuclein) is the key protein in neurodegenerative pathologies such as Parkinson's disease[21]. Although many lines of evidence suggested that Snca is involved in synaptic plasticity and learning[22- 
24], the physiological role of this protein in neurons still remains elusive[25]. Interestingly, in the cerebellum, Snca is particularly enriched in the vestibulocerebellum, a region that is associated with the vestibular and oculomotor functions[26]. Our study revealed not only an upregulation of Snca, but also an upregulation of Sncb ( $\beta$-synuclein), a highly homologous isoforms of Snca, at $24 \mathrm{hrs}$ after OKR-up learning. Therefore, the elevation of both Snca and Sncb after OKR-up learning may exhibit a correlation between synaptic modification in the cerebellar flocculus and LTM of OKR-up.

Trim9 (TRIpartite Motif 9) is a brain-enriched E3 ubiquitin ligase [27, 28] but also a regulator of morphogenesis in neurons[29, 30]. Trim 9 knockout mice show severe deficits of dendritic arborization in the hippocampus, and spatial learning and memory[31].

Adnp (Activity-dependent neuroprotective protein) is related to chromatin function and transcription[32,33]. Adnp is enriched in the hippocampus and cerebellum[34]. Haploinsufficiency of Adnp in mice causes a significant reduction of dendritic spine density in hippocampal and cortical neurons, and alteration of synaptic gene expression[35]. The increase in Adnp may reflect the possibility that STM of OKR-up may be associated with changes in dendritic spine density or synaptic gene expression in cerebellar neurons.

\section{Supplemental References}

1. Pfaffl, M.W., A new mathematical model for relative quantification in real-time RT-PCR. Nucleic Acids Res, 2001. 29(9): p. e45.

2. Foskett, J.K., et al., Inositol trisphosphate receptor Ca2+ release channels. Physiol Rev, 2007. 87(2): p. 593-658.

3. Furuichi, T., et al., Widespread expression of inositol 1,4,5-trisphosphate receptor type 1 gene (Insp3r1) in the mouse central nervous system. Receptors Channels, 1993. 1(1): p. 1124.

4. Inoue, T., et al., Type 1 inositol 1,4,5-trisphosphate receptor is required for induction of longterm depression in cerebellar Purkinje neurons. Journal of Neuroscience, 1998. 18(14): p. 5366-5373.

5. Pratt, K.G., et al., Presenilin 1 regulates homeostatic synaptic scaling through Akt signaling. Nat Neurosci, 2011. 14(9): p. 1112-4.

6. Zhang, C., et al., Presenilins are essential for regulating neurotransmitter release. Nature, 2009. 460(7255): p. 632-6.

7. Takami, K., et al., Expression of presenilin-1 and -2 mRNAs in rat and Alzheimer's disease brains. Brain research, 1997. 748(1-2): p. 122-130.

8. Cesca, F., et al., The synapsins: key actors of synapse function and plasticity. Prog Neurobiol, 2010. 91(4): p. 313-48.

9. Dorgans, K., et al., Short-term plasticity at cerebellar granule cell to molecular layer interneuron synapses expands information processing. Elife, 2019. 8.

10. Reim, K., et al., Complexins regulate a late step in Ca2+-dependent neurotransmitter release. Cell, 2001. 104(1): p. 71-81.

11. Rizo, J. and T.C. Sudhof, Snares and Munc18 in synaptic vesicle fusion. Nat Rev Neurosci, 
2002. 3(8): p. 641-53.

12. Gibson, H.E., et al., A similar impairment in CA3 mossy fibre LTP in the R6/2 mouse model of Huntington's disease and in the complexin // knockout mouse. Eur J Neurosci, 2005. 22(7): p. 1701-12.

13. Glynn, D., R.A. Bortnick, and A.J. Morton, Complexin // is essential for normal neurological function in mice. Hum Mol Genet, 2003. 12(19): p. 2431-48.

14. Harrison, P.J. and S.L. Eastwood, Preferential involvement of excitatory neurons in medial temporal lobe in schizophrenia. Lancet (London, England), 1998. 352(9141): p. 1669-1673.

15. Curmi, P.A., et al., The stathmin/tubulin interaction in vitro. J Biol Chem, 1997. 272(40): p. 25029-36.

16. Cassimeris, L., The oncoprotein 18/stathmin family of microtubule destabilizers. Curr Opin Cell Biol, 2002. 14(1): p. 18-24.

17. Grenningloh, G., et al., Role of the microtubule destabilizing proteins SCG10 and stathmin in neuronal growth. J Neurobiol, 2004. 58(1): p. 60-9.

18. Shumyatsky, G.P., et al., stathmin, a gene enriched in the amygdala, controls both learned and innate fear. Cell, 2005. 123(4): p. 697-709.

19. Ohkawa, N., et al., The microtubule destabilizer stathmin mediates the development of dendritic arbors in neuronal cells. J Cell Sci, 2007. 120(Pt 8): p. 1447-56.

20. Wang, W., et al., Distinct cerebellar engrams in short-term and long-term motor learning. Proc Natl Acad Sci U S A, 2014. 111(1): p. E188-93.

21. Waxman, E.A. and B.I. Giasson, Molecular mechanisms of alpha-synuclein neurodegeneration. Biochim Biophys Acta, 2009. 1792(7): p. 616-24.

22. Keri, S., et al., \{alpha\}-Synuclein gene duplication impairs reward learning. Proc Natl Acad Sci U S A, 2010. 107(36): p. 15992-4.

23. Liu, S., et al., alpha-Synuclein produces a long-lasting increase in neurotransmitter release. EMBO J, 2004. 23(22): p. 4506-16.

24. Durante, V., et al., Alpha-synuclein targets GluN2A NMDA receptor subunit causing striatal synaptic dysfunction and visuospatial memory alteration. Brain, 2019. 142(5): p. 1365-1385.

25. Burre, J., M. Sharma, and T.C. Sudhof, Cell Biology and Pathophysiology of alpha-Synuclein. Cold Spring Harb Perspect Med, 2018. 8(3).

26. Lee, S.K., et al., alpha-Synuclein expression in the mouse cerebellum is restricted to VG/UT1 excitatory terminals and is enriched in unipolar brush cells. Cerebellum, 2015. 14(5): p. 51627.

27. Berti, C., et al., TRIM9 is specifically expressed in the embryonic and adult nervous system. Mechanisms of Development, 2002. 113(2): p. 159-162.

28. Tanji, K., et al., TRIM9, a novel brain-specific E3 ubiquitin ligase, is repressed in the brain of Parkinson's disease and dementia with Lewy bodies. Neurobiol Dis, 2010. 38(2): p. 210-8. 
29. Winkle, C.C., et al., A novel Netrin-1-sensitive mechanism promotes local SNARE-mediated exocytosis during axon branching. J Cell Biol, 2014. 205(2): p. 217-32.

30. Menon, S., et al., The E3 Ubiquitin Ligase TRIM9 Is a Filopodia Off Switch Required for Netrin-Dependent Axon Guidance. Dev Cell, 2015. 35(6): p. 698-712.

31. Winkle, C.C., et al., Trimg Deletion Alters the Morphogenesis of Developing and Adult-Born Hippocampal Neurons and Impairs Spatial Learning and Memory. J Neurosci, 2016. 36(18): p. 4940-58.

32. Dresner, E., et al., Novel evolutionary-conserved role for the activity-dependent neuroprotective protein (ADNP) family that is important for erythropoiesis. J Biol Chem, 2012. 287(48): p. 40173-85.

33. Ostapcuk, V., et al., Activity-dependent neuroprotective protein recruits HP1 and CHD4 to control lineage-specifying genes. Nature, 2018. 557(7707): p. 739-743.

34. Gozes, I., Activity-dependent neuroprotective protein: from gene to drug candidate. Pharmacol Ther, 2007. 114(2): p. 146-54.

35. Hacohen-Kleiman, G., et al., Activity-dependent neuroprotective protein deficiency models synaptic and developmental phenotypes of autism-like syndrome. J Clin Invest, 2018. 128(11): p. 4956-4969. 
A

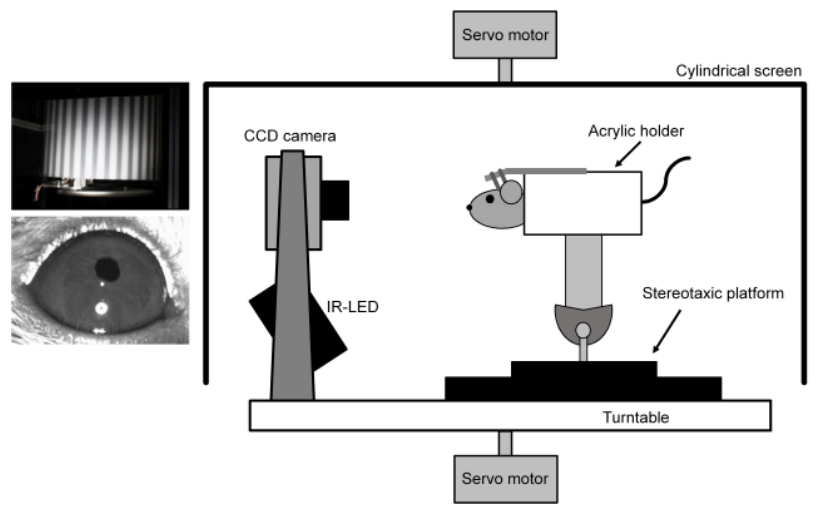

B

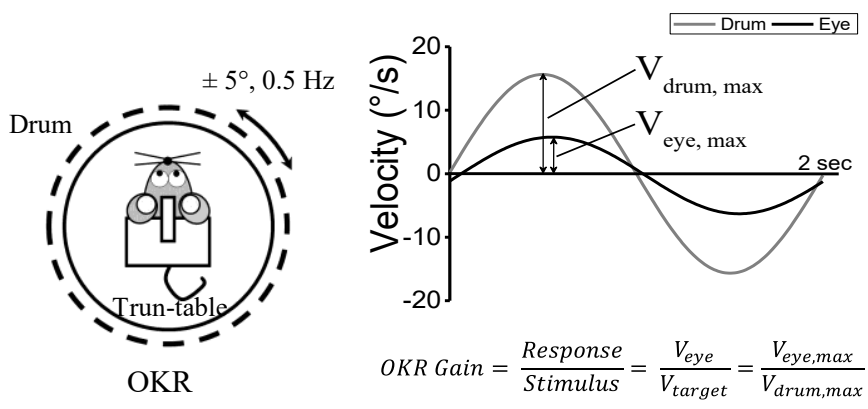

C
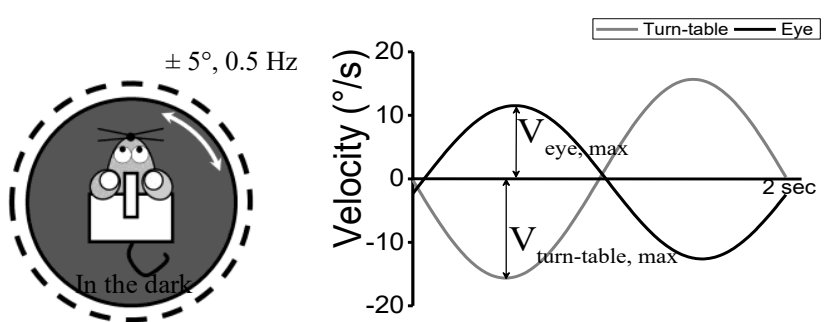

VOR

VOR Gain $=\frac{\text { Response }}{\text { Stimulus }}=\frac{V_{\text {eye }}}{V_{\text {head }}}=\frac{V_{\text {eye, } \max }}{V_{\text {turn }} \text {-table, } \text { max }}$

Supplemental Figure S1. Oculomotor test in mice. (A) Custom-made apparatus for behavioral tests. left: two photos showing frontal view of the apparatus (top) and videooculography (bottom). right: schematic drawing of the apparatus. (B) left: optokinetic response (OKR). In our experiment, OKR was induced by sinusoidal oscillation of the screen with amplitude of $\pm 5^{\circ}$ and frequency of $0.5 \mathrm{~Hz}$. right: OKR gain. OKR gain is defined as a ratio of the eye velocity to the screen velocity. (C) left: vestibulo-ocular reflex (VOR). VOR was evoked by sinusoidal oscillation of the turntable with amplitude of $\pm 5^{\circ}$ and frequency of $0.5 \mathrm{~Hz}$ in the dark. right: VOR gain. VOR gain is defined as a ratio of the eye velocity to the turntable velocity. 


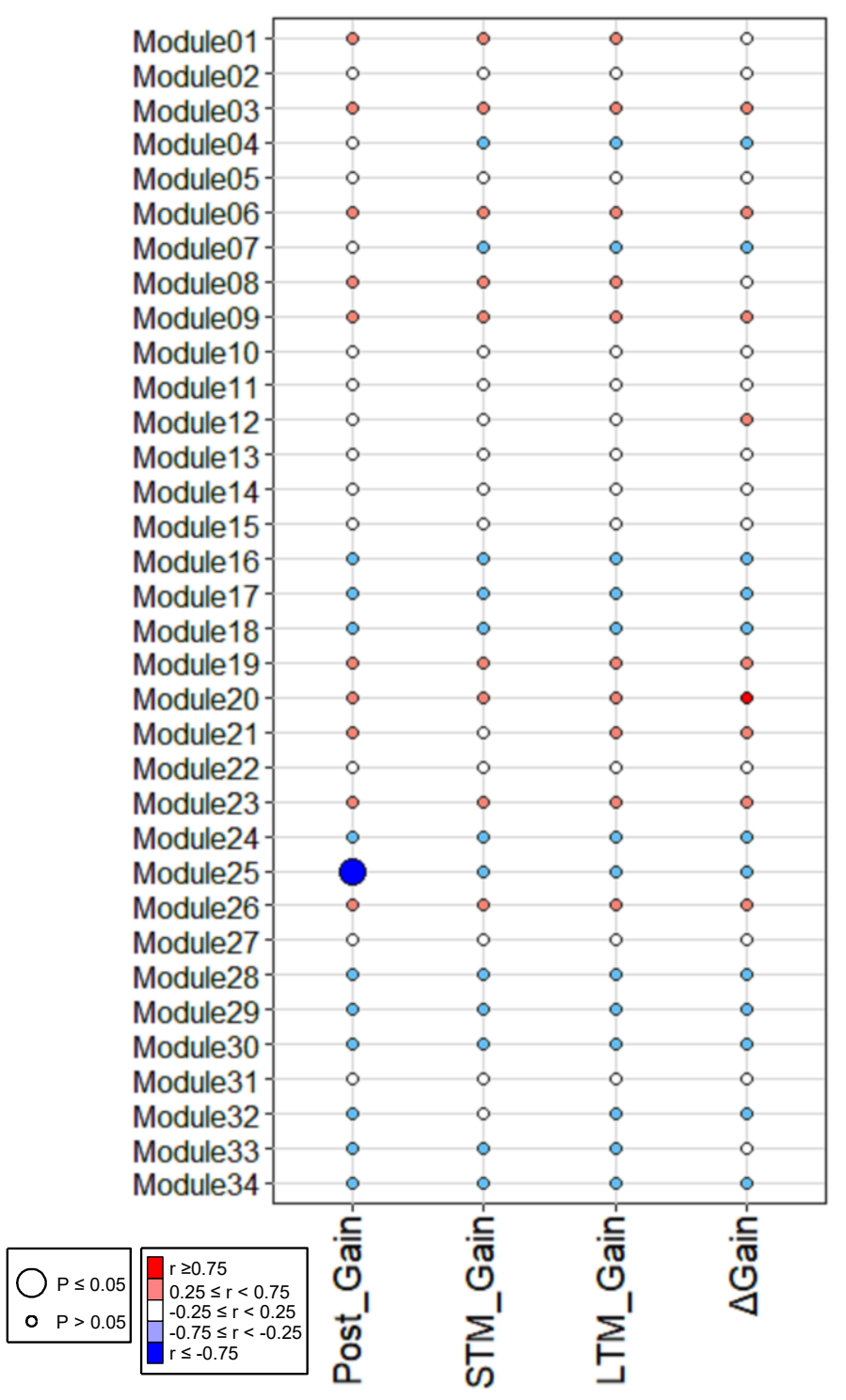

Supplemental Figure S2. Module-VOR memory relationships. Each row corresponds to a module. Each column corresponds to four behavioral traits, Post-training gain, STM gain, LTM gain, and $\Delta$ gain. In the bubble chart, each cell contains the corresponding correlation coefficients between module and behavioral trait, and their p-values. 


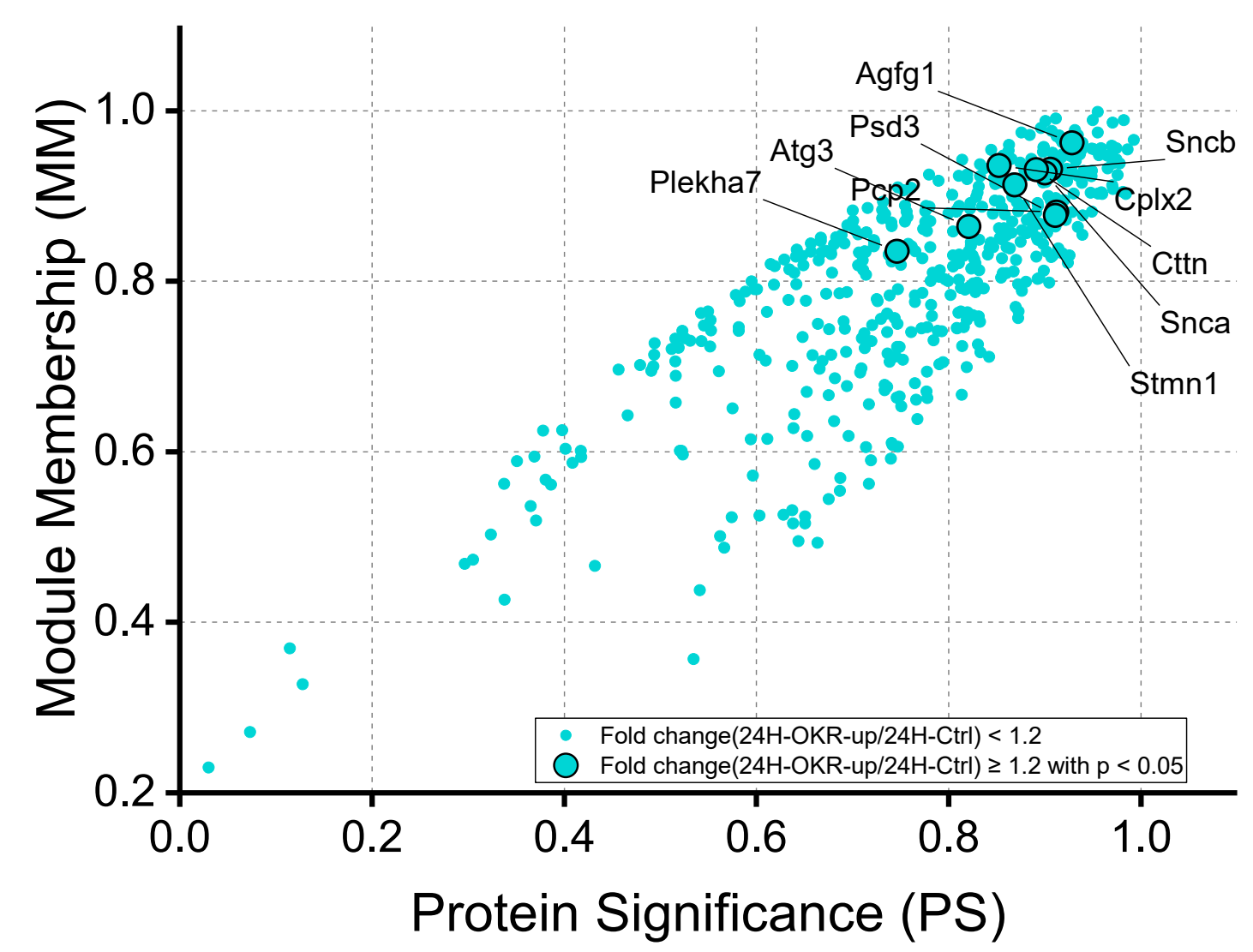

Supplemental Figure S3. Intramodular analysis of Module32 in WGCNA. In Module32, module membership (MM) and protein significance (PS) exhibit a strong positive correlation $(r=0.71, p=0.01)$, implying that the most important proteins in the module (proteins with a high MM) also tend to be highly correlated with $\Delta$ Gain of OKR learning (high position in PS). Of 486 proteins in this module, ten proteins exhibited a fold change greater than 1.2 (relative to the control group) with $p$-value $\leq$ 0.05 (bigger circles). 


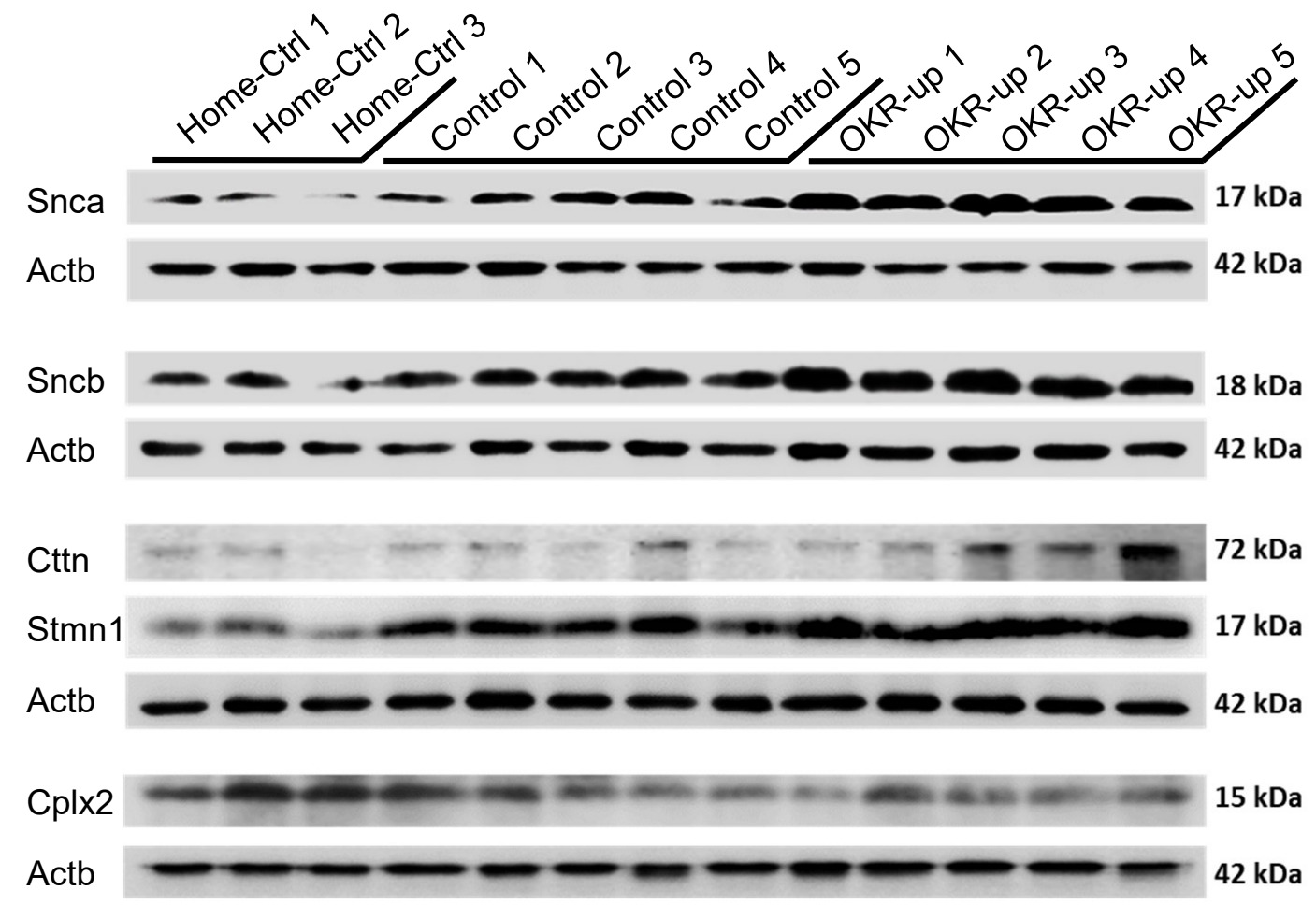

Supplemental Figure S4. Western blotting raw data of Figure 7. 
A

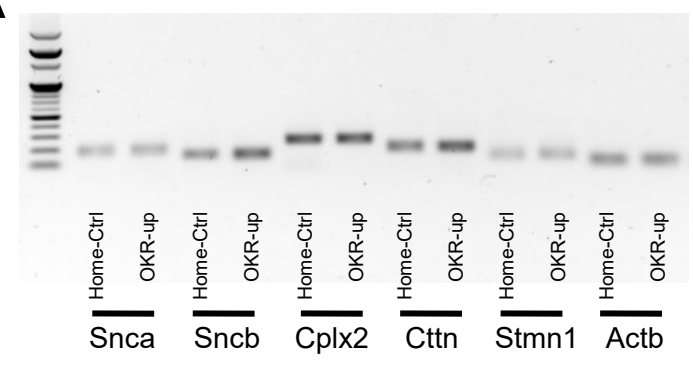

B

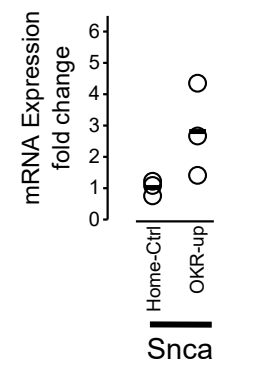

○

O

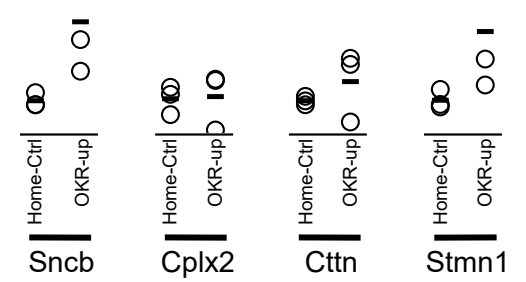

Supplemental Figure S5. RT-qPCR Validation of selected proteins. (A) RT-qPCR analysis of Snca, Sncb, Cplx2, Cttn, Stmn1, and Actb mRNA in cerebellar flocculus from Home-Ctrl and OKR-up groups ( $\mathrm{n}=3$ mice per group). (B) Quantification of the ratio of each gene/Actb ( $\mathrm{n}=3$ per group). Each ratio and mean value within a group are displayed as open circle and horizontal bar, respectively. 
A

B
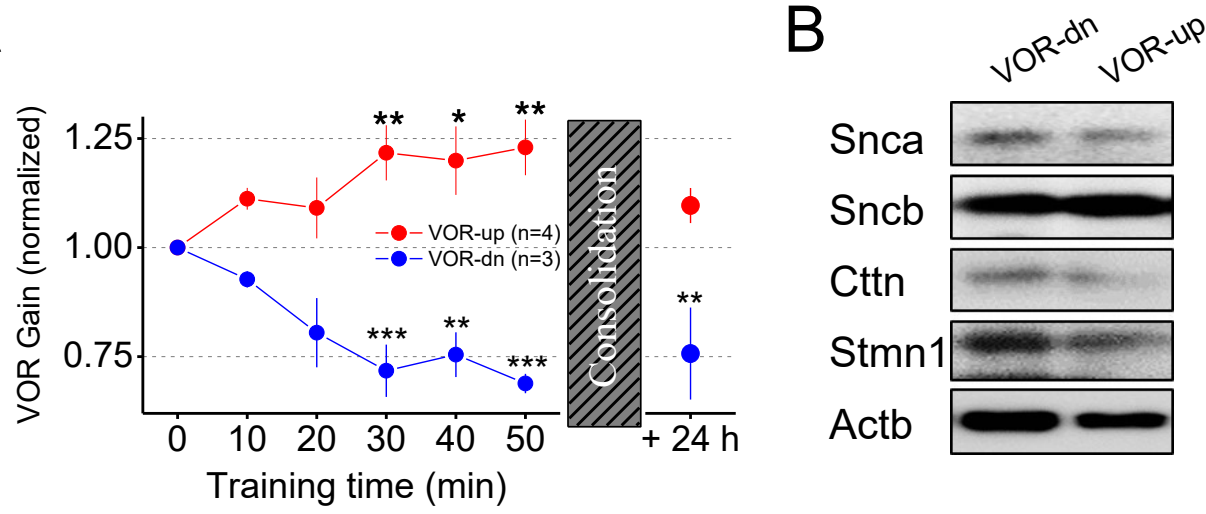

C

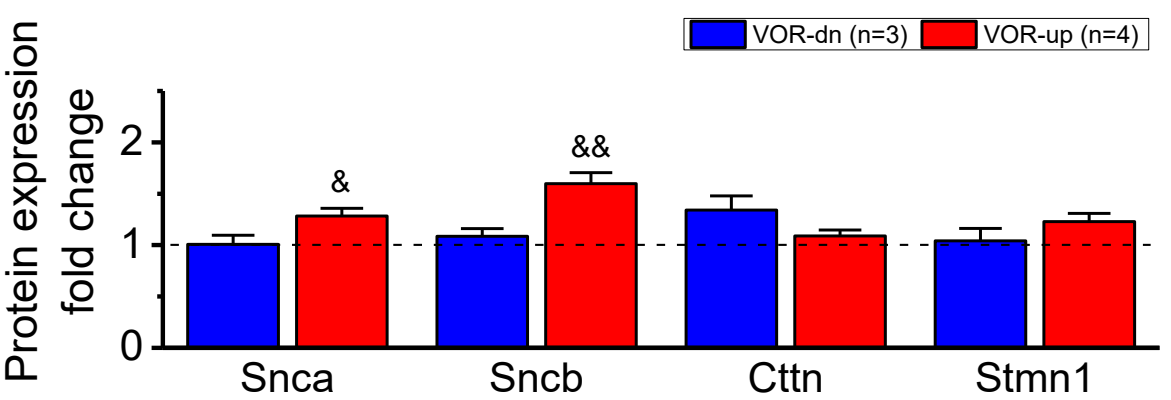

Supplemental Figure S6. Biological validation of selected proteins for the sample from VOR learning groups with memory consolidation of 24 hours. (A) Changes in VOR gain of VOR-dn $(n=3)$ and VOR-up $(n=4)$ groups during training and consolidation session. Linear mixed model post hoc Tukey test used for testing statistical significance between 0 min (pretraining) and each check point. $* \mathrm{p}<0.05, * * \mathrm{p}<0.01$ and $* * * \mathrm{p}<0.001$. (B) Representative Immunoblotting bands of 5 proteins (Snca, Sncb, Cttn, Stmn1, and Actb). (C) Averaged results of immunoblotting in VOR-dn (blue, $n=3$ ) and VOR-up (red, $n=4$ ). Averaged expression of each protein in Home-Ctrl $(n=5)$ display as dashed line. Error bars denote the SEM. Oneway ANOVA post hoc Bonferroni-test was used for comparisons of mean difference among VOR-dn, VOR-up, and Home-Ctrl groups. \& p $<0.05$, $\& \& \mathrm{p}<0.01$ between Home-Ctrl and VOR-up group. Data express mean \pm SEM throughout the figure. 


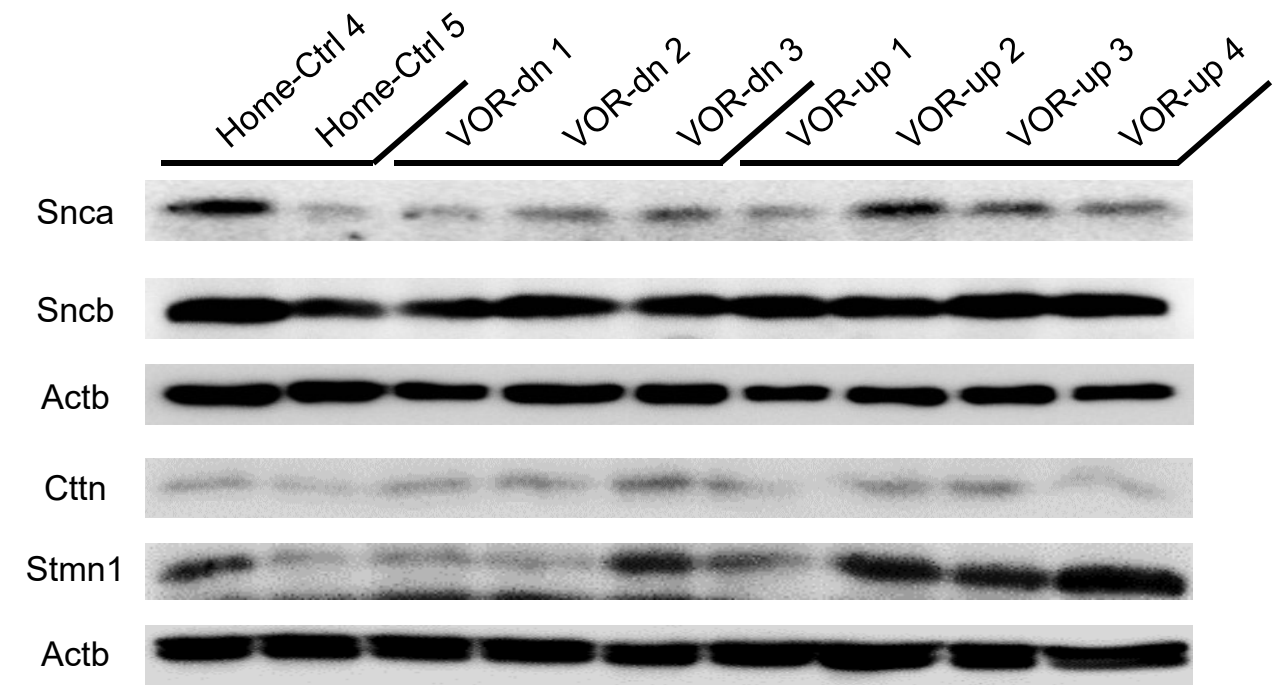

Supplemental Figure S7. Western blotting raw data of Supplemental Figure S6. 